



\section{I Z I O N A R I O B I O G R A F I C O DEGLI ITALIANI}


(C)

PROPRIETÀ ARTISTICA E LETTERARIA RISERVATA ISTITUTO DELLA ENCICLOPEDIA ITALIANA FONDATA DA GIOVANNI TRECCANI S.p.A.

2017

ISBN 978-88-12-00032-6

Stampato in Italia - Printed in Italy

Stamperia Artistica Nazionale S.p.A. - Trofarello (Torino)

2017 


\title{
ENCICLOPEDIA ITALIANA
}

\author{
FONDATA DA GIOVANNI TRECCANI
}

\author{
PRESIDENTE \\ FRANCO GALLO
}

\section{CONSIGLIO DI AMMINISTRAZIONE \\ VICEPRESIDENTI \\ MARIO ROMANO NEGRI, GIOVANNI PUGLISI}

LUIGI ABETE, PAOLO AIELLI, DOMENICO ARCURI, GIAMPIETRO BRUNELLO, MASSIMILIANO CESARE, PIERLUIGI CIOCCA, MARCELLO CLARICH, GIOVANNI

DE GENNARO, DANIELE DI LORETO, MATTEO FABIANI, LUIGI GUIDOBONO CAVALCHINI GAROFOLI, MASSIMO LAPUCCI, MONICA MAGGIONI, MARIO NUZZO,

GUIDO GIACOMO PONTE, GIANFRANCO RAGONESI, FRANCESCO VENOSTA

\section{DIRETTORE GENERALE}

\author{
MASSIMO BRAY
}

\section{COMITATO D’ONORE}

GIULIANO AMATO, FRANCESCO PAOLO CASAVOLA, FABIOLA GIANOTTI, TULLIO GREGORY, GIORGIO NAPOLITANO, PIETRO RESCIGNO

\section{CONSIGLIO SCIENTIFICO}

ENRICO ALLEVA, ANNA AMATI, LINA BOLZONI, IRENE BOZZONI, GEMMA CALAMANDREI, SILVIA CANDIANI, LUCIANO CANFORA, ENZO CHELI, MICHELE CILIBERTO, ESTER COEN, ELENA CONTI, SAMANTHA CRISTOFORETTI, JUAN CARLOS DE MARTIN, LUDOVICO EINAUDI, AMALIA ERCOLI FINZI, LUCIANO FONTANA, RENZO GATTEGNA, EMMA GIAMMATTEI, CARLO GUELFI, FERNANDO MAZZOCCA, MARIANA MAZZUCATO, MELANIA G. MAZZUCCO, ALBERTO MELLONI, ALESSANDRO MENDINI, DANIELE MENOZZI, ENZO MOAVERO MILANESI, CARLO MARIA OSSOLA, MIMMO PALADINO, GIORGIO PARISI, TERESA PÀROLI, GIANFRANCO PASQUINO, GILLES PECOUT, ALBERTO QUADRIO CURZIO, LUCA SERIANNI, SALVATORE SETTIS, GIANNI TONIOLO, VINCENZO TRIONE, CINO ZUCCHI

\section{COLLEGIO SINDACALE}





\title{
DIZIONARIO BIOGRAFICO DEGLI ITALIANI
}

\author{
DIRETTORE SCIENTIFICO
}

Raffaele Romanelli

\section{COMITATO DI DIREZIONE SCIENTIFICA}

Lorenzo Bianconi, Francesco Caglioti, Michele Ciliberto, Pietro Corsi, Alberto Melloni, Amedeo Quondam, Gian Maria Varanini, Maria Antonietta Visceglia

con la collaborazione della Fondazione per le Scienze Religiose Giovanni XXIII, Bologna, e della Scuola Normale Superiore, Pisa

\section{CONSULENTI SCIENTIFICI}

Franco Amatori, Antonio Clericuzio, Maria Conforti, Vito Loré, Berardo Pio

\section{DIREZIONE EDITORIALE}

\section{REDAZIONE ENCICLOPEDICA}

RESPONSABILE: Monica Trecca

Lavorazione e revisione dei testi: Lucia Armenante

Segreteria: Patrizia Morchio, Giancarla Palumbo, Silvia Zuccarello

\section{PRODUZIONE E ATTIVITÀ TECNICO-ARTISTICHE}

\section{ART DIRECTOR: Gerardo Casale}

Pianificazione e Produzione: Gerardo Casale; Antonella Baldini, Graziella Campus Magazzino: Fabrizio Izzo

Segreteria: Carla Proietti Checchi

Hanno contribuito con un servizio editoriale adHoc srl (Lulli Bertini, Gianni Bovini, Simone Caputo, Riccardo D’Anna, Antonella Dentamaro, Michele Di Sivo, Sara Esposito, Federica Favino, Gianluca Forgione, Daniele Frison, Gian Luca Fruci, Umberto Longo, Manuela Maggi, Maria Isabella Marchetti, Riccardo Martelli, Gregorio Moppi, Eloisa Mura, Franco Pignatti, Luigi Russo, Floriana Santini, Laura Schettini, Michela Tarallo, Valeria Tavazzi, Paola Vinesi, Federico Vizzaccaro) e per l'impaginazione Alborada srl 



\section{Collaboratori del novantesimo volume}

Luca Addante, Sammarco Ottavio, Sanseverino Ferrante; Guillaume Alonge, Sanseverino Federico, Sanseverino Galeazzo, Sanseverino Gaspare, detto Capitan Fracassa; Elisa Andretta, Salviani Ippolito, Santo Mariano; Giuseppe Astuto, San Giuliano Antonino Paternò Castello marchese di; Luca Aversano, San Martino Valperga Enrico, conte di Maglione; Massimo Baioni, Sauro Nazario; Giovanna Baldissin Molli, Sansone Francesco; Roberto Balzani, Savini Savino; Matteo Baragli, Santucci Carlo; Andrea Bartocci, Sampieri Floriano, Sangiorgi Giovanni, Sangiorgi Giovanni Antonio; Antonella Barzazi, Sarpi Paolo (Pietro); Nadia Bastogi, Santi di Tito (Santi Titi, Santi di Tito Titi); Maria Beltramini, Sanmicheli (da San Michele, Sanmichele, da San Michiel) Michele; Michèle Benaiteau, Sanseverino Niccolò Berardino, Sanseverino Pietrantonio, Sanseverino Roberto; Elisabetta Benucci, Sarchiani Giuseppe; Francesco Bertola, Santini Giovanni Sante Gaspero; Frida Bertolini, Salvioli, Gaetano; Paola Bianchi, San Martino della Motta Felice; Silvestra Bietoletti, Santarelli Emilio; Italo Birocchi, Satta Salvatore (Giovanni Salvatore); Silvia Blasio, Salvi Giovanni Battista, detto il Sassoferrato; Paola Bonani, Sanfilippo Antonio; Maddalena Bonechi, Saracini Claudio; Annamaria Bonsante, Sassano Matteo; Jean-Paul Boyer, Sancia di Maiorca, regina di Sicilia-Napoli; Matteo Brera, Sanesi Roberto; Luca Brogioni, Sansoni Giulio Cesare; Bella Brover-Lubovsky, Sarti Giuseppe Francesco Eligi; Giampiero Brunelli, Savelli Antonello, Savelli Fabrizio, Savelli Giulio, Savelli Giulio, Savelli Paolo, Savelli Paolo Francesco, Savelli Silvio; Francesca Brunet, Salvotti Antonio; Moreno Bucci, Sarri Luigi Egisto; Dario Busolini, Savelli Camilla Virginia; Alviera Bussotti, Santucci Loreto; Claudio Caldarazzo, Santasofia Giovanni, Santasofia Marsilio; Federica Camata, Saporiti Teresa; Michele Camerota, Santillana Giorgio Diaz (de); Jean-André Cancellieri, Sambucuccio di Alando; Rossella Cancila, Santa Croce Pietro Celeste (Celestre, Celestri) marchese di, Santa Lucia Francesco Branciforte Barresi duca di; Salvatore Canneto, Santinelli Francesco Maria; Alessandra Capanna, Samonà Giuseppe; Alessandro Capone, Savelli Domenico; Valerio Caprara, Satta Flores Stefano; Simone Caputo, Sassi Gianni; Irene Maria Caraba, Saratelli Giuseppe Giacomo; Allì Caracciolo, Salviati Filippo Vincenzo Romolo; Diego Carnevale, Sarcone Michele, Savaresi (Savarese) Antonio (Mario Timoleone); Laura Carotti, Sancassani Dionisio Andrea; Martina Carraro, Sardi Giovanni; Mirco Carrattieri, Saragat Giuseppe; Pinuccia Carrer, Sartori Claudio Giulio Maria; Francesco Carta, Salvetti Angelo; Cesarina Casanova, Sassatelli Giovanni; Michele Cassese, Sartori Luigi; Francesco Casula, Satta Sebastiano; Raffaella Catini, Salvi Gaspare, Sant'Elia Antonio, Sardi Giuseppe, Sarti Antonio, Sartoris Alberto; Michela Catto, Sassi Giuseppe Antonio; Silvano Cavazza, Santonino Paolo; Giovanni Cecini, Santoni Ermenegildo; Francesco Paolo de Ceglia, Santucci Antonio, delle Pomarance (Ripomarance); Riccardo Chellini, Sanzanome (Senzanome); Adriana Chemello, Sanvitale Thiene Leonora; Giampaolo Chillè, Samperi Placido; Maria Conforti, Santucci Bernardo; Elisabetta Corsi, Sambiase (Sambiasi, Sanbiase) Francesco; Pasquale Corsi, Sanseverino Ruggero; Pietro Corsi, Savi Gaetano, 
Savi Paolo; Valerio Corvisieri, Sargentini Luisa (Luisa Spagnoli); Danilo Costantini, Sanseverino Aurora; Paolo Cova, Santi Giovanni; Daniele Cozzoli, Sanarelli Giuseppe; Vittoria Crespi Morbio, Sanquirico Alessandro; Emanuele Curzel, Sartori Giovanni (Giovanni Maria); Paolo Da Col, Santarelli Santi Giuseppe; Alessandro D’Alfonso, Sanmartino Giuseppe; Fabio D’Angelo, Sangiovanni Giosuè; Leandra D’Antone, Saraceno Pasquale; Antonino De Francesco, Santarosa Filippo Annibale Santorre De Rossi conte di, Sappia Enrico, Sauli Gaspare; Davide De Franco, San Martino di Parella Alessio Maurizio, San Martino di Parella Carlo Ludovico Emilio; Piero Del Negro, Sauget Roberto de; Paolo Delorenzi, Santi Sebastiano; Pier Paolo De Martino, Salviucci Giovanni; Stefano De Mieri, Santafede Fabrizio; Renato Di Benedetto, Saponaro Giacomo; Massimo Di Gioacchino, Satolli Francesco (in religione Francesco di Paola); Francesco Paolo Di Teodoro, Santi (Sanzio) Raffaello; Ugo Dovere, Sanfelice d'Acquavella Guglielmo, Sanseverino Gaetano; Piergiorgio Dragone, Savelli Angelo; Enrico Dumas, Sassone di Anagni; Teresa D’Urso, Sanvito Bartolomeo; Anna Esposito, Santacroce Andrea, Santacroce Giorgio, Santacroce Prospero; Davide Esposito, Sanguinacci Jacopo; Maria Cecilia Fabbri, Salvestrini Bartolomeo; Francesca Fantappiè, Saracinelli Ferdinando; Andrea Fara, Sauli Bendinelli (Bendinello, Bandinello), Sauli Bendinelli (Bendinello, Bandinello); Vincenzo Farinella, Santi (Sanzio) Raffaello; Federica Favino, Santini Antonio; Lucia Felici, Sartori Giovanni Leonardo (Giovanni Leone Nardi, Giovanni Leonardi, Giovanni Leonardo Sertori); Liliana Ferrari, Santin Antonio; Cesare Fertonani, Sammartini Giovanni Battista, Sammartini Giuseppe (Gioseffo Francesco Gaspare Melchiorre Baldassarre); Pietro Finelli, Savio Bartolomeo (Bartolomeo Francesco Savi); Vittoria Fiorelli, Sambuca Giuseppe Beccadelli di Bologna e Gravina marchese della; Fiorenzo Fisogni, Savanni (Savani) Francesco; Emanuele Fontana, Sangiorgio Martino da; Liletta Fornasari, Santini Bernardino; Federico Fornoni, Salvi Matteo; Irene Fosi, Savelli Federico, Savelli Giacomo, Savelli Onorio, Savelli Paolo, Savelli Troilo; Gigliola Fragnito, Sanseverino Barbara; Franco Franceschi, Sapori Armando; Gian Luca Fruci, Saracco Giuseppe; Laura Gaffuri, Savelli (Giordani de Insula) Giovanni; Cristina Galassi, Salvucci Matteuccio; Andrea Garavaglia, Santo Pietro (San Piè, San Pier, San Piero, Sampier, Sampietro) De’ (del, di) Negri (Negro) Giulio, Sartorio (Sertorio) Antonio; Marina Gazzini, Sanvitale Obizzo (de Sancto Vitale, Obitius, Obizo, Opiço); Piergiovanni Genovesi, Sanvitale Jacopo, Sanvitale Luigi; Marco Gentile, Sanvitale Gilberto; Massimo Gentili-Tedeschi, Sartori Claudio Giulio Maria; Adriano Ghisetti Giavarina, Sangallo (da Sangallo) Bastiano, detto Aristotile, Sangallo (da Sangallo) Giovan Francesco; Gabriele Giacomelli, Sanmartini (Sammartini) Piero (Pietro); Massimo Giansante, Samaritani Ranieri; Claudio Gigante, Salviati Lionardo; Silvano Giordano, Santacroce Andrea, Sasso Lucio, Sauli Antonio Maria; Francesco Giuntini, Salvi Antonio; Federico Alessandro Goria, San Giorgio Giacomino da (Giacomino de Michelonibus); Alberto Gottarelli, Sanseverino Vimercati Alfonso; Alessandro Grandolfo, Santacroce Girolamo, Sarti Andrea; Antoine-Marie Graziani, Sampiero (Gian Piero) di Bastelica (trad. dal francese di Marco Lapenna); Francesco Guidi Bruscoli, Sassetti Francesco; Giuseppe Gullino, Sanudo (Sanuto) Benedetto, Sanudo (Sanuto) Federico, Sanudo (Sanuto) Livio; Pierre Hurtubise, Salviati Alamanno, Salviati Antonio Maria, Salviati Bernardo, Salviati Gregorio Antonio Maria (traduzioni dal francese di Marco Lapenna); Frédéric Ieva, Sanvitale Fortunino; Girolamo Imbruglia, Sangro Raimondo di; Marco Impiglia, Santoni Achille (Achille Michele Angelo, Achille Michelangelo); Christine Jeanneret, Sarti Giuseppe Francesco Eligi; Alexander Koller, Santacroce Ottavio; Emmanuel Lamouche, Sanquirico Paolo; Giancarlo Landini, Salvi Lorenzo, Sammarco Mario; Marco Leone, Sarnelli Pompeo; Lucilla Lijoi, Savarese Antonino (Nino); Francesco Li Pira, Sangineto; Stefano L'Occaso, Samacchini Orazio; Francesco Lora, Sandoni Pietro (Pier) Giuseppe; Niva Lorenzini, Sanguineti Edoardo; Filippo Lovison, Sauli Domenico; Sergio Lubello, Salvioni Carlo; Guido Lucchini, Sapegno Natalino; Francesco Lucioli, Sandei (Sandeo) Ludovico; Flavia Luise, Santa Elisabetta Antonino Montaperto e Massa duca di; Ausilia Magaudda, Sanseverino Aurora; Massimo Malinverni, Sasso Panfilo; Anton Giulio Mancino, Samperi Salvatore; Marco Manfredi, Sanminiatelli Cosimo Andrea; Luca Marcelli, Savi Domenico; Cristiano Marchegiani, Salvi Nicola (Niccolò), Sambonet Roberto, Sanfelice Ferdinando; Stefano 
Margutti, Sanvitali Federico; Federica Marinoni, Sanesi Ireneo; Paola Marongiu, Salvetti Maddalena; Francesco Martelli, Salviati Maria; Silvia Massari, Sarti Carlo, Sarti Lorenzo; Benedetta Matucci, Salvini Salvino, Santarelli Giovanni Antonio; Igor Melani, Sassetti Tommaso; Matteo Melchiorre, Sanudo (Sanuto) Marino (Marin) il Giovane; Guido Melis, Sandulli Aldo Mazzini; Raffaele Mellace, Sarro (Sarri, Sarra) Domenico Natale; Sabrina Minuzzi, Saraceno Marino; Pietro Misuraca, Savagnone Giuseppe; Maddalena Moglia, Saporito (Sapollitus, Saporitus, Savoritus) Guglielmo; Giorgio Montecchi, Sassuolo Manfredino da; Gregorio Moppi, Santoliquido Francesco; Maria Teresa Mori, Salvo Muzio Rosa (Rosina); Leonardo Morlino, Sartori Giovanni; Emiliano Morreale, Sampietro Ines Isabella (Isa Miranda); Eloisa Mura, Satta Salvatore (Giovanni Salvatore); Stefano Musso, Santi Fernando; Stefania Nanni, Sanna Demetrio; Paola Nardone, Saquella; Simona Negruzzo, Sanvitale Antonio Francesco, Sardagna Carlo Emanuele von Hohenstein; Gerardo Nicolosi, Sauli Francesco Maria; Sandro de Nobile, Santi Piero; Elisa Novi Chavarria, Sanfelice Gennaro, Sanfelice Giovanni Francesco, Sanfelice Giovanni Tommaso, Sanfelice Giovanni Vincenzo, Sanfelice Giuseppe; Biagio Nuciforo, Sanseverino Ruggero I, Sanseverino Ruggero II; Sara Orfali, Salviati Andrea, Salviati Forese, Salviati Francesco, Salviati Jacopo; Fabrizio Orsini, Santelli Italo e Giorgio; Nicolai E. Østenlund, Sarti Giuseppe Francesco Eligi; Anna Ottani Cavina, Saraceni Carlo; Alessandro Ottaviani, Savelli Roberto; Serena Pacchiani, Santagata Antonio Giuseppe; Jacopo Paganelli, San Girolamo Andrea da; Nicla Palladino, Sannia Achille; Italo Pantani, Sardi Ludovico; Maria Pia Paoli, Salvini Anton Maria, Salvini Salvino; Riccardo Parmeggiani, San Girolamo Andrea da; Renato Pasta, Santi Giorgio; Michele Pellegrini, Sansedoni Ambrogio, beato; Chiara Petrolini, Sarotti Paolo; Serena Pezzini, Sarrocchi Margherita; Annalisa Pezzo, Sartorio Giulio Aristide; Roland Pfeiffer, Sarti Giuseppe Francesco Eligi; Gregorio Piaia, Santinello Giovanni; Filippo Piazza, Sandrini Tommaso; Marco Pierini, Sarrocchi Tito; Pietro Pietrini, Sarteschi Pietro Paolo; Berardo Pio, Sangro Gentile di; Eleonora Plebani, Savelli Bernardino; Alma Poloni, Sampante Ranieri, Sancasciano da; Valentina Puleo, Santucci Luigi; Blythe Alice Raviola, San Giorgio Giovanni Francesco Biandrate di; Roberto Regoli, Sanseverino Stanislao, Santucci Vincenzo; Saverio Ricci, Santori (Santoro, Santorio) Giulio Antonio; Paola Ricco, Savioli Leonardo; Francesca Romana Rietti, Sapelli Luigi (in arte Caramba); Laura Righi, Sanuti Nicolosa (nata Castellani); Paolo Alberto Rismondo, Sances (Sanci, Sonzi, Sonci, Sanchez) Giovanni Felice; Alessandra Rizzi, Sanudo (Sanuto) Marino il Vecchio; Enrico Rogora, Sansone Giovanni; Laura Ronchi, Sanudo Vincenzo (in religione Pietro Aurelio); Anna Maria Rossetti, Sarti Emiliano; Edoardo Rossetti, Sangiorgio Giovanni Antonio; Luca Rossetto, Sandi Vettore Felice; Paolo Rosso, Sandigliano Guglielmo; Giancarlo Rostirolla, Santini Fortunato; Alessio Russo, Sanseverino Antonello, Sanseverino Antonio, Sanseverino Girolamo (Geronimo), Sanseverino D’Aragona Roberto; Renzo Sabbatini, Sardi Cesare, Sardi Cesare Giuseppe, Sardini Giacomo, Sardini Giovan Battista Domenico, Sardini Scipione; Marco Sabbioneti, SantoroPassarelli Francesco; Rodolfo Sacchettini, Santuccio Giovanni (Gianni); Guillaume Saint-Guillain, Sanudo (Sanuto) Marco I, duca dell'Arcipelago; Dora Sallay, Sano di Pietro; David Salomoni, Sanvitale Gianquirico, Sanvitale Pietro Brunoro; Daniele Sanguineti, Santacroce; Elisabetta Sarmati, Samonà Carmelo; Orietta Sartori, Santi Leone, Savioni Mario; Giuseppe Sava, Sartori Antonio Giuseppe; Raffaele Savigni, Sandonnini Nicola; Nicola Scaldaferri, Sassu Pietro Costantino; Gabriele Scalessa, Sani Luigi, Sassernò Agathe Sophie; Marco Scansani, Savelli Sperandio (Sperandio da Mantova); Laura Schettini, Sambri Vittorina; Mirella Schino, Salvini Tommaso; Raffaella Seligardi, Salvigni Pellegrino; Jean Sénié, Santacroce Prospero (trad. dal francese di Valerié Duhautoy); Marica Setaro, Sanvenero Rosselli Gustavo; Gerardo de Simone, Sanguigni Battista di Biagio; Marcello Simonetta, Salviati Giovanni, Salviati Jacopo, Sanga Giovan Battista; Lorenzo Sinisi, Saredo Giuseppe; Giulio Sodano, Sanseverino Lucio, Saraceni (Saracino) Giovanni Michele; Bruna Soravia, Santillana David (de); Marta Stefani, Sangallo (San Gallo, San Galli, Sangalli) Pietro Paolo da; Nico Stringa, Santomaso Giuseppe; Francesco Surdich, Sangermano Vincenzo, Sapeto Giuseppe, Sassetti Filippo, Savio Pietro; Stefano Tabacchi, San Marzano Carlo Emanuele, San Marzano Filippo Antonio; Alberto Tanturri, Santacroce Antonio; Walter Tega, Santucci Antonio; Paolo Teofilatto, Santini Paolo; 
Rodobaldo Tibaldi, Savioli Alessandro; Franco Tomasi, Sanguinacci Scipione; Luca Tonetti, Santorini Giovanni Domenico; Luca Torre, Sandoval De Castro Diego; Stefania Torre, Savarese Giacomo, Savarese Roberto; Elisabetta Torselli, Santini Gabriele, Sanzogno Giovanni Giuseppe Luigi, detto Nino; Giovanna Tosatti, Savini Nicci Oliviero; Giuseppe Trebbi, Santorio Santorio; Luigi Tufano, Sanseverino Giovanni, Sanseverino Ugo, Sanseverino D'Aragona Giovan Francesco; Maurizio Ulturale, San Germano Pietro da, Saracino Andreotto (Andreotto di Saracino Caldera); Giovanna Uzzani, Sassu Aligi; Gian Maria Varanini, San Bonifacio (Sambonifacio); Marcella Varriale, Sangiovannara (Marianna De Crescenzo); Paolo Varvaro, Sansanelli Nicola; Ignazio Veca, Salviati Scipione Maria; Carlo Vecce, Sannazaro Iacopo; Marco Vendittelli, Sant'Eustachio, Savelli Giacomo, Savelli Luca, Savelli Pandolfo; Paola Venturelli, Saracchi; Monica Venturini, Sanvitale Francesca, Sapienza Goliarda; Natale Vescio, Salvioli Giuseppe; Stefano Villani, Salvetti Antelminelli Giovanni; Fabrizio Vistoli, Savignoni Luigi; Paola Volpini, Salviati Vincenzo (Vincenzio); Giulia Zaccariotto, Savin Paolo; Gilda Zazzara, Santarelli Enzo; Guido Zucconi, Sardi Giovanni.

Il profilo culturale del DBI è nel suo lemmario, nelle forme che esso è andato assumendo nel tempo. La preparazione di una Biografia nazionale, "monumento storico dell'identità civile» del Paese fu avviata nel 1925 e affidata da Giovanni Gentile a Fortunato Pintor (1877-1960). Nei decenni seguenti una schiera di redattori raccolse circa 400.000 schede su circa 200.000 personaggi dal V al XX secolo. Quando l'opera iniziò a pubblicarsi, fu deciso che avrebbe menzionato circa 40.000 biografie e gli altri nomi sarebbero poi confluiti in un apposito Repertorio, che non fu mai fatto. Da allora e per molti anni i redattori hanno via via composto i vari volumi sulla base dello schedario esistente. Nella primavera del 2010 è stata definita una proposta per l'intero lemmario, prevedendo la conclusione dell'opera per l'anno 2020, e con il volume centesimo. Sotto la guida dell'allora direttore Mario Caravale, il compito è stato svolto da Gabriella Bartolini, Alessandra Bonfigli, Alessandra Cimmino, Fulvio Conti, Riccardo D’Anna, Stefania De Guzzis, Michele Di Sivo, Federica Favino, Simona Feci, Simona Foà, Paola Longobardi, Giuseppe Monsagrati, Claudia Montuschi, Arnaldo Morelli, Francesco Mozzetti, Paola Pietrini, Franco Pignatti, Federico Pirani, Renato Sansa, Maria Giovanna Sarti, Sonia Springolo, Alessandra Uguccioni, Maria Antonietta Visceglia. Successivamente, sotto la direzione di Raffaele Romanelli e di un apposito comitato scientifico, è iniziata una riflessione sui diversi caratteri che nel frattempo ha assunto l'identità civile della quale si stava erigendo il monumento. Con l'ausilio di consulenti e delle maggiori società professionali nei diversi settori, il lemmario ha preso l'attuale forma e viene costantemente riplasmato seguendo il procedere degli studi e delle conoscenze. Oggi consta di 40.928 voci, delle quali 34.911 già pubblicate, su carta o on-line. 


\section{AVVERTENZE}

Le voci segnalate con la dizione «v. www.treccani.it» sono pubblicate nella versione on-line dell'opera, contenuta nel portale Treccani.

Tutte le biografie hanno come esponente il cognome del personaggio, con le eccezioni seguenti:

a) quando si tratti di santi (i beati, invece, sono collocati sotto il cognome, ove lo si conosca). Es.: GIOVANNI Bosco, santo (ma ALBERTONI, Ludovica [beata]).

b) quando si tratti di papi.

c) quando si tratti di re e di personaggi che abbiano esercitato la sovranità

(e le rispettive consorti), mentre il nome di famiglia serve da discriminante alfabetico.

Es.: ALESSANDRO Farnese, duca di Parma e Piacenza ALESSANDRO de' Medici, duca di Firenze.

Entrano in questa categoria i detentori dei seguenti titoli: conti, poi duchi di Savoia, re di Sardegna, re d'Italia marchesi, poi duchi di Monferrato

duchi di Milano

marchesi, poi duchi di Mantova

duchi di Parma e Piacenza

duchi di Modena e Reggio e Ferrara

duchi di Firenze e granduchi di Toscana

duchi di Urbino

re di Sicilia, di Napoli, delle Due Sicilie.

I membri non regnanti di queste famiglie e i sovrani delle altre dinastie italiane sono da ricercare sotto i nomi di famiglia.

d) quando si tratti del nome di religione degli appartenenti ai seguenti Ordini religiosi:

cappuccini

carmelitani scalzi

eremitani scalzi di S. Agostino

passionisti

recolletti di S. Agostino

trinitari.

Per gli agostiniani, e in taluni casi per i minori, oscillando nella documentazione e nella tradizione storiografica la forma del nome, le biografie sono svolte sotto il cognome (ove questo sia in uso) oppure sotto il nome di religione. 
Per i nobili piemontesi e siciliani le biografie sono svolte prevalentemente sotto il nome del feudo principale.

Per i cognomi preceduti dalle particelle prepositive semplici e articolate $d i, d e$ ', dei, degli, ecc., si distinguono i casi seguenti:

a) i cognomi che solo l'uso locale ha reso composti con la particella prepositiva (es.: degli Abati, degli Albizzi, de’ Medici, ecc.) sono registrati sotto il semplice cognome.

\section{Es.: ABATI, ALBIZZI, MEDICI.}

b) i cognomi che, al tempo in cui viveva il personaggio biografato, avevano già assunto come parte integrante la particella prepositiva sono registrati sotto la lettera D.

\section{Es.: DEL GIUDICE, DELLA CORTE, DELLA TOSA.}

Nei casi in cui il Dizionario ha adottato una forma tra due o più ugualmente diffuse nella tradizione e nell'uso viene fatto un rinvio.

I personaggi femminili vanno ricercati sotto il cognome da nubile, con l'eccezione di quei casi già sopra contemplati (sante, regine, ecc.).

\section{Italianizzazione}

Per il carattere prevalentemente storico dell'opera, non si è proceduto all'italianizzazione sistematica dei nomi, ma ci si è attenuti all'uso prevalente.

Es.: ACTON, Guglielmo (in quanto effettivamente italianizzato) ACTON, John Francis Edward.

Successione alfabetica degli esponenti

Per la successione alfabetica vale l'esponente in neretto. Un esponente composto di più elementi in neretto è considerato come un'unica parola. Si avrà quindi la seguente successione:

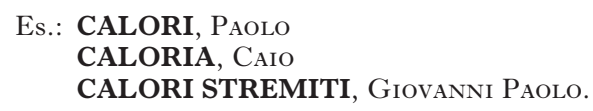

Dopo il neretto, la discriminante grafica è rappresentata dal MAIUSCOLETTO, indipendentemente da qualsiasi particella prepositiva che possa unire i due elementi. Si avrà la seguente successione: nome, nome + toponimo o soprannome; cognome, nome.

\section{Es.: CORRADO, marchese di Monferrato \\ CORRADO CONFALONIERI, santo \\ CORRADO di MARLENHEIM \\ CORRADO, CORRADINO.}

All'interno di una serie di omonimi il criterio della successione è rappresentato dall'ordine cronologico.

Es.: ABBAti, Pietro Paolo, il Vecchio

ABBATI, Pietro Paolo, il Giovane.

All'interno di una serie di omonimi, costituiti da un solo esponente, potranno darsi casi del tipo seguente:

\section{BENEDETTO... \\ BENEDETTO I, papa.}

Con l'aggiunta dell'ordinale le serie dei papi non vengono interrotte e si facilita la ricerca. 
I santi, che vengono registrati sotto il nome (es.: GIOVANNI BOSCO, santo), quando abbiano l'esponente costituito soltanto da esso (es.: BERNARDO, santo), sono inseriti nella serie degli omonimi, secondo un criterio meramente cronologico.

Gli ordinali (I, II, III, IV, ecc.) sono riservati, nell'esponente, solo ai papi e ai sovrani. In tutti gli altri casi gli ordinali sono soppressi nell'esponente, ma adoperati nel corpo della voce biografica.

Rinvii

a) Il rinvio è, in generale, semplice.

\section{Es.: TRIONFO, Agostino: v. Agostino d'Ancona.}

Per distinguere i casi di omonimia, il rinvio sarà corredato da brevi indicazioni.

Es.: ANTONiO da Ferrara: v. Alberti, Antonio, pittore, secc. XIV-XV.

ANTONIO da Ferrara: v. Beccari, Antonio, poeta, sec. XIV.

b) Nei casi di varianti grafiche di alcuni cognomi, usate indifferentemente nelle opere storiche, si adotta all'inizio di ogni singola serie un rinvio generico alle altre.

Es.: ABATE: v. anche Abbate, Abati, Abbati, ecc.

ABBATE: v. anche Авate, Abati, Авbati, ecc.

ABBATI: v. anche Aвате, Авati, Abвate, ecc.

Nell'esponente, fra parentesi, si registrano, accanto alla grafia adottata dal Dizionario, quelle forme che, pur non essendo prevalenti, sono comunque attestate.

Es.: BETTO (Berto) di Geri.

c) Per le donne il rinvio al cognome da nubile è adottato solo in quei casi particolari in cui ciò possa più facilmente far individuare il personaggio.

Es.: GARIBALDi, Anita: v. Ribeiro da Silva, Anita.

d) Per i nobili piemontesi e siciliani che vengano svolti sotto il nome del feudo viene fatto un rinvio specifico dal cognome: LANZA e STELLA, Pietro, principe di Trabia: v. Trabia, Pietro Lanza e Stella principe di.

e) Per i nobili non contemplati in $d$ ) che siano più comunemente noti col nome del feudo, viene fatto un rinvio specifico dal nome del feudo:

Es.: Macchia, Gaetano Gambacorta principe di: v. Gambacorta, Gaetano.

f) Per gli pseudonimi non è fatto rinvio, essendo implicito che tutte le voci siano svolte sotto il vero nome. Qualche eccezione è fatta per quei personaggi il cui pseudonimo prevale nell'uso.

Es.: CONTESSA LARA: v. Cattermole, Eva.

g) Per gli umanisti viene fatto un rinvio dal nome umanistico.

POliziano: v. Ambrogini, Angelo.

Il nome umanistico compare come esponente soltanto nel caso in cui non sia stato possibile accertare quello di famiglia.

ABBREVIAZIONI

$\mathrm{PG}=$ Patrologia Greca

$\mathrm{PL}=$ Patrologia Latina

$\mathrm{MGH}=$ Monumenta Germaniae Historica

$\mathrm{RIS}=$ Rerum Italicarum Scriptores 

D I Z I O N A R I O

B I O G R A F I C O

DEGLI ITALIANI 

$\mathrm{XC}$

SALVESTRINI - SAVIOZZO DA SIENA 
Il volume è stato chiuso in redazione nel mese di ottobre 2017. 